\title{
Rifaximina nel trattamento della malattia diverticolare: potenziale terapeutico ed economico
}

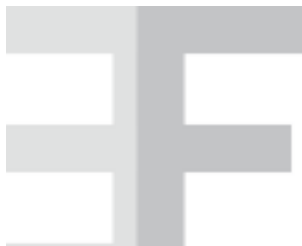

Orietta Zaniolo*, Mario Eandi*

PROFILO

\begin{abstract}
Antibiotics are commonly used to treat major inflammatory complications of diverticular disease, but apparently there is no rationale for the use of antibiotic therapy in uncomplicated disease, where an inflammatory component is by definition excluded.

Some observations suggest a possible role of gut microflora in determining some symptoms related to diverticular disease: bacterial overgrowth, amplifying gas production and bacterial degradation of fibres, could determine bloating, pain and high fecal density. Therefore a beneficial antibiotic action on diverticular disease symptoms can be hypothesized.

In this paper pharmacokinetic, pharmacodinamic and therapeutic potential of an intestinal antibiotic, rifaximin, are reviewed.

Rifaximin is a rifamycin derivative which acts by inhibiting bacterial ribonucleic acid (RNA) synthesis. It is virtually unabsorbed after oral administration and in vitro data indicate it to possess a broad spectrum of action; bacterial resistance during exposure to rifaximin has been reported but its clinical importance remains to be fully defined.

The results of placebo-controlled clinical trials show that cyclic administration of rifaximin is more effective in reducing symptoms and in preventing complications than fibre supplementation alone; the drug appears to be well tolerated and safe.

We consider the clinical implications and economical impact of diverticulosis on the Italian National Health System and the patient, with a particular attention on the cost of hospitalization, surgery and global management of diverticulitis and other common disease complications.

The hypothetic savings correlated to the reduction of complications incidence, attainable with rifaximin use have been calculated and the comparison between the different acquisition costs of the drug frequently used to treat the diverticular disease is provided.

Finally we reviewed some quality of life trials in which the psychological and sociological influence of the disease symptoms and treatments on the patient are assessed.
\end{abstract}

Keywords: diverticular disease, rifaximin, antibiotics, economical impact

Farmeconomia e percorsi terapeutici 2005; 6 (1): 5-20

\section{INTRODUZIONE}

Il termine "malattia diverticolare" si riferisce a un concetto clinico usato a proposito di pazienti affetti da diverticolosi, sia sintomatica che asintomatica [1]. La diverticolosi del colon è una patologia acquisita caratterizzata dalla formazione di un gran numero di estroflessioni sacciformi della parete dell'intestino, localizzate più frequentemente nel colon sinistro, e in particolare nel colon sigmoideo.

Queste erniazioni, dette appunto diverticoli, sono in genere causate da un'esagerata pressione all'interno del lume intestinale (diverticolo da pulsione) o, in casi limitati, da un' aderenza infiammatoria tra un organo cavo e linfonodi vicini (diverticolo da trazione). Si ritiene attualmente che l'alta frequenza di questa patologia sia almeno in parte il risultato di un'alimentazione povera di fibre e, in generale, dello stile di vita moderno.

La malattia può dare una sintomatologia acuta o cronica. Nella prima i sintomi che compaiono più frequentemente sono: dolore al quadrante addominale inferiore sinistro (diverticolite del sigma) o periombelicale (diverticolite digiunale), alternanza di stitichezza e diarrea, anoressia e, frequentemente, sangue nelle feci, febbre e vomito; in quella cronica i sintomi sono gli stessi, ma attenuati e ricorrenti nel tempo.

Quando si verifica un'infiammazione il quadro prende il nome di diverticolite; questa è la complicanza che si manifesta più frequentemen-
*Farmacologia Clinica, Università di Torino 
te: la comparsa di episodi ricorrenti si osserva nel 7-35\% dei pazienti e, una volta superato il primo attacco, il rischio annuale che si ripeta è del 3\% [2,3]. Il meccanismo e la causa per cui si instaura il processo flogistico non sono ancora del tutto chiari, ma un cambiamento della microflora intestinale o una crescita eccessiva di quest'ultima potrebbero rappresentare una delle spiegazioni più plausibili [4].

L'infiammazione può propagarsi e determinare complicazioni ancora più gravi; in rari casi una sacca infiammata o infetta può rompersi, rovesciando il suo contenuto di scarti intestinali all'interno della cavità peritoneale: la peritonite che ne consegue richiede un intervento chirurgico tempestivo.

Nel 10-30\% dei pazienti con diverticolite può manifestarsi una proctorragia variabilmente significativa sul piano clinico, mentre la comparsa di emorragia grave è limitata al 3-5\% dei casi.

Altre complicazioni della diverticolite possono includere la formazione di un ascesso o una fistola. L'incidenza di fistolizzazione è variabile, però raggiunge la massima frequenza nei pazienti più anziani con una più lunga storia di diverticolosi alle spalle; quelle che insorgono più comunemente sono le fistole colovescicali.
Il tessuto cicatriziale formatosi in seguito all'infezione cronica del colon può portare a ostruzione parziale o totale del grosso intestino, rendendo spesso necessario l'intervento chirurgico [5]. Ciascuna delle complicazioni più comuni della diverticolite (perforazione, ostruzione, emorragia, fistole) si verifica nel 5-15\% circa dei pazienti a un follow up tra 10 e 30 anni [6].

Nei casi in cui la terapia farmacologica non è sufficiente ad alleviare i sintomi e a rimuovere l'infiammazione (se presente), viene asportato il diverticolo, in caso di formazione singola e di grosse dimensioni, o l'intero tratto interessato se sono presenti estroflessioni multiple.

Sono possibili due tipi di intervento chirurgico: la resezione segmentaria standard e la resezione dell'intestino con colostomia.

Nel primo caso vengono mantenuti i movimenti intestinali normali e, a seconda dell'entità dell'infiammazione, si può eseguire un intervento a cielo aperto o in laparoscopia; se invece l'infiammazione è talmente estesa da non poter permettere la successiva ricongiuntura di colon e retto, il colon è connesso allo stoma. La colostomia può essere temporanea o permanente.

Il trattamento della malattia diverticolare non complicata è generalmente di tipo medico, dato che la maggior parte dei pazienti recupera abbastanza velocemente senza provvedimenti

\begin{tabular}{|c|c|c|c|c|c|}
\hline Organismo & $\begin{array}{l}\mathrm{MIC}_{50} \mathrm{a} \\
(\mathrm{mg} / \mathrm{L})\end{array}$ & $\begin{array}{l}\mathrm{MIC}_{90} \mathrm{a} \\
(\mathrm{mg} / \mathrm{L})\end{array}$ & Organismo & $\begin{array}{l}M^{M I C} C_{50} a \\
(\mathrm{mg} / \mathrm{L})\end{array}$ & $\begin{array}{l}\mathrm{MIC}_{90} \mathrm{a} \\
(\mathrm{mg} / \mathrm{L})\end{array}$ \\
\hline Batteri Gram positivi & & & Batteri Gram negativi & & \\
\hline Staphylococci & & & Enterobacteriaceae & & \\
\hline S. aureus & 0.015 & $=<0.015$ & Citrobacter spp. & $>8$ & $>8$ \\
\hline S. aureus & $=<0.015$ & $>8$ & Enterobacter spp. & $>=4$ & $>=8$ \\
\hline \multirow[t]{2}{*}{ S. epidermidis } & $=<0.015$ & $=<0.015$ & Escherichia coli & $>=3.12$ & $>8$ \\
\hline & & & Klebsiella spp. & $>8$ & $>8$ \\
\hline Streptococci/enterococci & & & Proteus spp. & $>=4$ & $>=4$ \\
\hline Enterococcus faecalis & 2 & 8 & Salmonella spp. & $>=2$ & $>6.25$ \\
\hline Enterococcus faecium & 2 & $>8$ & Serratia spp. & $>=25$ & $>50$ \\
\hline \multirow[t]{2}{*}{ Enterococcus spp. } & 0.25 & 2 & Shigella spp. & 4 & 8 \\
\hline & & & Yersinia enterocolitica & $>=3$ & $>8$ \\
\hline \multicolumn{6}{|l|}{ Clostridia } \\
\hline C. difficile & 0.2 & 0.8 & Bacteroides fragilis & 0.1 & 12.5 \\
\hline \multirow[t]{2}{*}{ Clostridium spp. } & 0.4 & 50 & Bacteroides spp. & 0.1 & $50 ;=<4$ \\
\hline & & & Acinetobacter spp. & 2 & 4 \\
\hline Bacillus cereus & 0.06 & & Pseudomonas aeruginosa & 8 & $>8$ \\
\hline Peptococcus spp. & 0.1 & 3.1 & Fusobacterium spp. & 0.4 & 50 \\
\hline \multirow[t]{2}{*}{ Peptostreptococcus spp. } & 0.2 & 25 & Helicobacter pylori & 8 & 16 \\
\hline & & & Campylobacter jejuni & $>=6.25$ & $>100$ \\
\hline
\end{tabular}

$\mathrm{MIC}_{50}=$ la più bassa concentrazione di farmaco in grado di inibire il $50 \%$ dei ceppi testati;

$\mathrm{MIC}_{90}=$ la più bassa concentrazione di farmaco in grado di inibire il $90 \%$ dei ceppi testati

Tabella I

Attività antibatterica in vitro [18] 
chirurgici; le misure terapeutiche sono inizialmente mirate ad ottenere una condizione di "riposo" dell'intestino, risolvendo lo stato infettivo, le conseguenze della flogosi e prevenendo o limitando le complicanze.

Il paziente deve restare allettato ed evitare qualsiasi assunzione di cibo o liquidi per bocca, specialmente se sono presenti nausea, vomito o distensione addominale.

Per la cura di questa patologia è stato suggerito un ruolo terapeutico delle fibre: alcuni trial clinici controllati hanno dimostrato il beneficio ottenuto sul piano sintomatico dalla somministrazione di crusca e di altri agenti in grado di aumentare la massa fecale [7,8]; a fronte di questi primi risultati però non c'è stata la conferma da parte di altri studi svolti che, anzi, non hanno dimostrato nessuna differenza, in termini di outcome, tra l'utilizzo di tali sostanze e la somministrazione di placebo $[9,10]$.

Gli agenti spasmolitici sono abbondantemente usati nel trattamento sintomatico della patologia, anche se non ci sono dati attendibili che ne sostengano la reale efficacia.

Il trattamento con antibiotici è già in uso in tutti quei casi in cui è sospettata un infiammazione a livello diverticolare e, ovviamente, in tutti i casi di complicanze batteriche più gravi. Relativamente all'utilizzo della terapia antibiotica nella patologia non complicata, sono stati svolti numerosi trial clinici che hanno analizzato l'efficacia di antibiotici scarsamente assorbibili, cioè ad azione locale, rispetto al trattamento del paziente con supplemento di fibre: essi sembrano ridurre più drasticamente sintomatologia e rischio di complicanze.

La scelta dell'antibiotico dovrebbe essere attuata sulla base della flora fecale presente nel singolo caso, tenendo presente che spesso alla diverticolite del colon si associa una batteriemia. In questo caso è opportuno ricorrere ad un antibiotico ad ampio spettro somministrato per via sistemica, mentre la somministrazione di un enteroantibiotico locale è preferibile nella malattia diverticolare senza complicanze, in quanto permette di evitare i possibile effetti collaterali, indotti dall'uso di antibiotici sistemici [11].

Rifaximina è un derivato semisintetico della rifamicina di cui mantiene praticamente invariato lo spettro d'azione (che risulta così abbastanza ampio), che ha però sostanziali differenze dal punto di vista farmacocinetico: meno dell' $1 \%$ della dose somministrata per via orale passa nel circolo sistemico [12]. Oltre alla sua evidente efficacia nella cura di numerose patologie, fra cui enterocoliti ed encefalopatie epatiche, numerosi studi clinici hanno testato l'utilità dell'uso di rifaximina nel trattamento della malattia diverticolare sintomatica non complicata, dando risultati favorevoli.

\section{PROFILOFARMACOLOGICO-CLINICO}

\section{Attività antibatterica e fenomeni di resistenza}

In pazienti affetti da diverticolite, i patogeni isolati sono, in ordine decrescente di frequenza, i coliformi, il Bacillus fragilis e i clostridi.

La rifaximina, come le altre molecole appartenenti alla classe delle rifamicine, esplica la propria attività terapeutica bloccando la trascrizione da DNA a RNAm: la molecola crea un legame irreversibile con la subunità $\beta$ dell'enzima RNA polimerasi batterica DNA-dipendente, inattivandolo [13,14].

Le indagini eseguite sull'attività antibatterica di questo farmaco hanno dimostrato che esso possiede lo stesso ampio spettro d'attività della rifamicina: la sua azione battericida si esplica nei confronti di numerose specie batteriche gram-positive e gram-negative, sia aerobie che anaerobie [15].

Per quanto riguarda i batteri Gram positivi, appare evidente dalla Tabella I la suscettibilità al farmaco dello Staphylococcus aureus oxacillina-sensibile, mentre il ceppo oxacillinaresistente mostra un range di valori di MIC abbastanza eterogeneo. Il farmaco svolge un'attività eccellente contro lo Staphylococcus epidermidis e contro gli streptococci del gruppo A, B, C, F e G [16]. Il Clostridium difficile si è rivelato sensibile a rifaximina [17], così come il Moraxella catarrhalis, l'Haemophilus influenzae, il Bacillus cereus e l'Eubacterium [16].

L'attività antimicrobica di rifaximina si esplica anche verso una serie di agenti potenzialmente patogeni in grado di causare infezioni a livello del tratto genitale, fra cui alcuni ceppi di Bacteroides bivius-disiens, Gardnerella vaginalis, Haemophilus ducreyi e Neisseria gonorrhoeae [16].

La resistenza batterica verso rifampicina si instaura abbastanza rapidamente sia in vitro che in vivo [13]. Rifaximina sembra condividere questa caratteristica: infatti durante uno studio svolto su volontari sani $(800 \mathrm{mg} /$ die per cinque giorni) è stato registrato un tasso di resistenza compreso fra il 30-90\% in alcuni ceppi di Enterobacteriaceae, Enterococcus, Bacteroides, Clostridium e su alcuni tipi di cocci anaerobi [19]. Entro 1-2 settimane dall'interruzione del trattamento, sempre nello stesso studio, si è rilevata una diminuzione del tasso di resistenza, che ha raggiunto valori inferiori al 20\%; in generale, si è notato che i batteri anaerobi, specialmente quelli Gram negativi, impiegano più tempo a riacquistare la sensibilità rispetto ai batteri aerobi.

A circa tre mesi dalla fine del trattamento, alcune analisi hanno rilevato la completa assenza di ceppi resistenti; queste indicazioni 
potrebbero spiegare la scarsa resistenza manifestata in seguito a somministrazione di rifaximina nei casi di encefalopatia epatica e diverticoliti, dove il trattamento è ciclico.

La spiegazione di tale fenomeno è in parte attribuita al fatto che lo sviluppo di resistenza verso rifaximina segua un meccanismo diverso rispetto alla resistenza mediata da plasmidi, comunemente acquisita dai batteri nei confronti degli antibiotici aminoglicosidici; tale meccanismo è simile a quello che porta alla formazione di ceppi resistenti nei confronti della rifampicina e, cioè un' alterazione cromosomica a livello dell'RNA polimerasi DNA-dipendente, bersaglio dell' antibiotico [19].

\section{Proprietà farmacocinetiche}

Una dose di rifaximina somministrata oralmente viene assorbita, in assenza di lesioni gravi della mucosa intestinale, in misura minore all' $1 \%$ [15].

Le analisi del sangue, svolte nove ore dopo somministrazione orale di $400 \mathrm{mg}$ di rifaximina in nove volontari sani, non hanno rilevato nessuna traccia del farmaco, mentre una concentrazione compresa fra 2 e $5,5 \mu \mathrm{g} / \mathrm{L}$ è stata rilevata in altri nove volontari fino a quattro ore dopo l'assunzione di rifaximina; nelle urine delle 48 ore successive alla somministrazione, il farmaco è risultato presente in misura minore dello $0,025 \%$ della dose assunta [20].

Alcuni studi svolti su ratti hanno rilevato che un'ora dopo somministrazione orale di $100 \mathrm{mg} / \mathrm{kg}$ di rifaximina, il 78,8\% del farmaco era ancora presente a livello intestinale [21] e che, dopo somministrazione orale di $25 \mathrm{mg} / \mathrm{kg}$, il recupero totale del farmaco nel corso delle 72 ore seguenti è stato dello $0,29 \%$ nelle urine e del $53,4 \%$ nelle feci [22]. In un altro studio, il tasso di recupero totale nelle feci di ratto durante le 72 e le 168 ore seguenti la somministrazione orale di rifaximina $(100 \mathrm{mg} / \mathrm{kg})$ è stato rispettivamente di 73 e $96 \%$ [23].

Uno studio condotto su 39 pazienti affetti da diarrea del viaggiatore [24] ha valutato la concentrazione fecale di rifaximina in seguito alla sua somministrazione orale $(800 \mathrm{mg} /$ die per tre giorni): il primo giorno post-trattamento la concentrazione fecale media è risultata pari a $7,961 \mathrm{mg} / \mathrm{g}$ con riduzione graduale nei giorni seguenti. In base ai valori misurati gli autori sono giunti alla conclusione, in accordo con quanto rilevato da precedenti studi farmacocinetici [20,25-27], che la maggior parte del farmaco viene eliminata nelle feci; inoltre è stato rilevato che la concentrazione fecale di rifaximina nei cinque giorni successivi alla fine del trattamento è stata sempre superiore alla MICs delle specie batteriche isolate durante lo studio.
Questi dati confermano la scarsissima assorbibilità del farmaco; maggiori informazioni sulla possibile inattivazione gastrointestinale di rifaximina potrebbero migliorare ulteriormente le conoscenze sull'iter farmacocinetico di questa molecola.

\section{Profilo terapeutico}

\section{Efficacia clinica}

Nei pazienti affetti da diverticolosi la terapia antibiotica viene usata soprattutto nel trattamento delle complicanze e, apparentemente, non c'è una base razionale che indichi il suo utilizzo nella malattia diverticolare non complicata (con infiammazione esclusa per definizione).

Tuttavia l'infiammazione della mucosa è stata dimostrata istologicamente anche in pazienti asintomatici e alcuni disturbi legati a questa patologia sembrano indotti o peggiorati dalla microflora intestinale: il metabolismo batterico produce gas (soprattutto $\mathrm{H}_{2}$ e $\mathrm{CH}_{4}$ ) che probabilmente rappresenta la causa principale di fenomeni come la sensazione di gonfiore e di dolore a livello addominale (fenomeno dell' "air trapping"). La terapia antimicrobica, oltre ad attenuare questi sintomi, può, riducendo la degradazione svolta dai batteri nei confronti delle fibre, indurre un aumento della massa delle feci e renderle più morbide e leggere: la formazione di diverticoli e la loro infiammazione sono infatti sfavorite quando la pressione che agisce sulle pareti del colon è bassa [28,29].

Nel 1991 è stato svolto un primo studio in aperto al fine di valutare l'efficacia della rifaximina nel trattamento di pazienti affetti da diverticolosi con diverticolite del colon di entità ambulatoriale. Il requisito fondamentale per essere ammessi al trial era l'evidenza endoscopica di diverticoli multipli del colon con fenomeni di flogosi della mucosa confermati istologicamente; inoltre i pazienti dovevano avere avuto, nei tre mesi precedenti l'arruolamento, almeno due dei seguenti sintomi non spiegabili con altre patologie: leucocitosi, episodi ricorrenti di febbre, o diarrea della durata superiore a sette giorni.

Allo studio sono stati ammessi 20 pazienti, a cui sono stati somministrati $400 \mathrm{mg}$ di rifaximina due volte al giorno per 10 giorni e la valutazione dell'effetto terapeutico è stata effettuata sia in base ai sintomi riportati dai partecipanti, sia secondo parametri oggettivi valutati endoscopicamente ed istologicamente.

Alla fine della terapia, sintomi come nausea, dolore addominale spontaneo e alla palpazione sono risultati significativamente ridotti rispetto alle valutazioni pre-trattamento e i pazienti con ipertermia e leucocitosi hanno mostrato un netto miglioramento; secondo i reperti endoscopici, in 
seguito alla terapia l'edema, l'iperemia, le soffusioni emorragiche e la granulia diffusa della mucosa sono significativamente diminuiti, mentre la vasodilatazione e i segni di emorragia, valutati mediante biopsia al termine del trattamento, hanno mostrato una tendenza alla riduzione senza tuttavia raggiungere la significatività [11] .

Nel 1992 Papi e colleghi hanno condotto uno studio in aperto, controllato e randomizzato, al fine di valutare l'efficacia di rifaximina nel trattamento a lungo termine di 217 pazienti affetti da malattia diverticolare non complicata. Di questi, 107 sono stati randomizzati a ricevere $2 \mathrm{~g}$ al giorno di glucomannano e $400 \mathrm{mg}$ di rifaximina due volte al giorno per sette giorni ogni mese; 110 (gruppo di controllo) hanno ricevuto solo glucomannano [30].

Questa sostanza è un polisaccaride (rientra tra le fibre alimentari) che, assorbendo più di cento volte il suo volume in acqua, aumenta la massa della feci e le rende meno compatte regolandone il transito intestinale; nel caso di diverticolosi, l'utilizzo di una dieta ad alto contenuto di fibre viene generalmente consigliato, sebbene gli studi randomizzati non abbiano ottenuto risultati omogenei a proposito degli effetti benefici di crusca e affini sui sintomi di tale patologia $[31,32,7]$.

I pazienti ammessi allo studio sono stati valutati prima di iniziare il trattamento e con cadenza bimestrale mediante l'assegnazione di uno score sintomatologico. Nel corso dello studio, durato 12 mesi, tale punteggio è diminuito in maniera significativamente maggiore nei pazienti che assumevano rifaximina: riduzione del

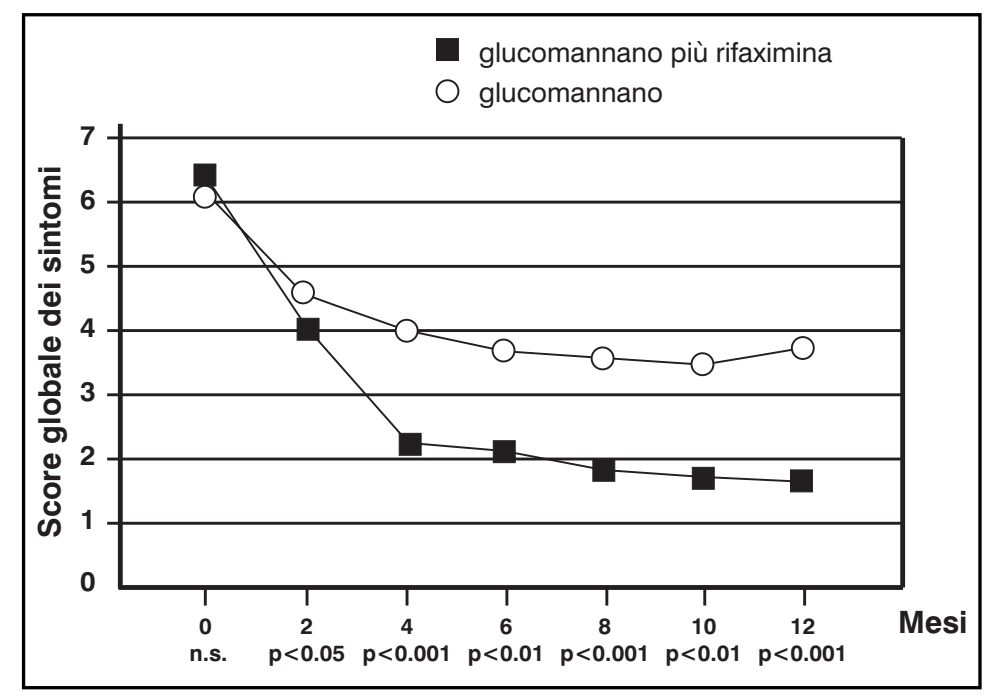

Figura 1

Andamento dello score globale nei due gruppi [30]

$63,9 \%$ nel gruppo trattato contro riduzione del $47,6 \%$ nel gruppo controllo (Figura 1).

Tali risultati sono stati confermati qualche anno dopo, sempre da Papi e colleghi, mediante uno studio randomizzato e controllato, svolto in doppio cieco e comprendente 168 pazienti [33]. Essi sono stati divisi in due gruppi, uno ricevente solo glucomannano $(\mathrm{n}=84)$ e l'altro glucomannano più rifaximina $(n=84)$, secondo lo schema posologico precedente per la durata di 12 mesi.

Entrambi i trattamenti sono stati efficaci nel ridurre lo score sintomatologico globale durante i primi tre mesi di trattamento, ma i pazienti trattati con rifaximina hanno rilevato una

\begin{tabular}{|c|c|c|}
\hline & Gruppo A & Gruppo B \\
\hline Trattamento & $\begin{array}{c}\text { rifaximina } \\
\text { (400 mg/bid per } 7 \text { giorni/mese) } \\
+ \\
\text { glucomannano }(4 \mathrm{~g} / \mathrm{die})\end{array}$ & glucomannano (4 g/die) \\
\hline Popolazione (ITT) & 595 & 373 \\
\hline Abbandono dello studio & $37(6,2 \%)$ & $27(7,2 \%)$ \\
\hline Popolazione (PP) & 558 & 346 \\
\hline \multicolumn{3}{|c|}{ RISULTATI dopo 12 mesi trattamento } \\
\hline Non compliance & 1 & 1 \\
\hline Score globale (ITT) & $\begin{array}{c}1,0 \pm 0,9 \\
(6,5 \pm 0,8 \text { al baseline })\end{array}$ & $\begin{array}{c}2,0 \pm 1,1 \\
(6,3 \pm 0,9 \text { al baseline })\end{array}$ \\
\hline Asintomatologia & $56,5 \%$ & $29,2 \%$ \\
\hline COMPLICANZE & $8(1,34 \%)$ & $12(3,22 \%)$ \\
\hline $\begin{array}{l}\text { Di cui: } \\
\text { Diverticolite } \\
\text { Sanguinamento rettale }\end{array}$ & $\begin{array}{l}6 \\
2\end{array}$ & $\begin{array}{r}11 \\
1\end{array}$ \\
\hline
\end{tabular}

Tabella II

Caratteristiche dello studio e suoi principali risultati

$\mathrm{ITT}=$ Intent-to-treat $\mathrm{PP}=$ per protocol 


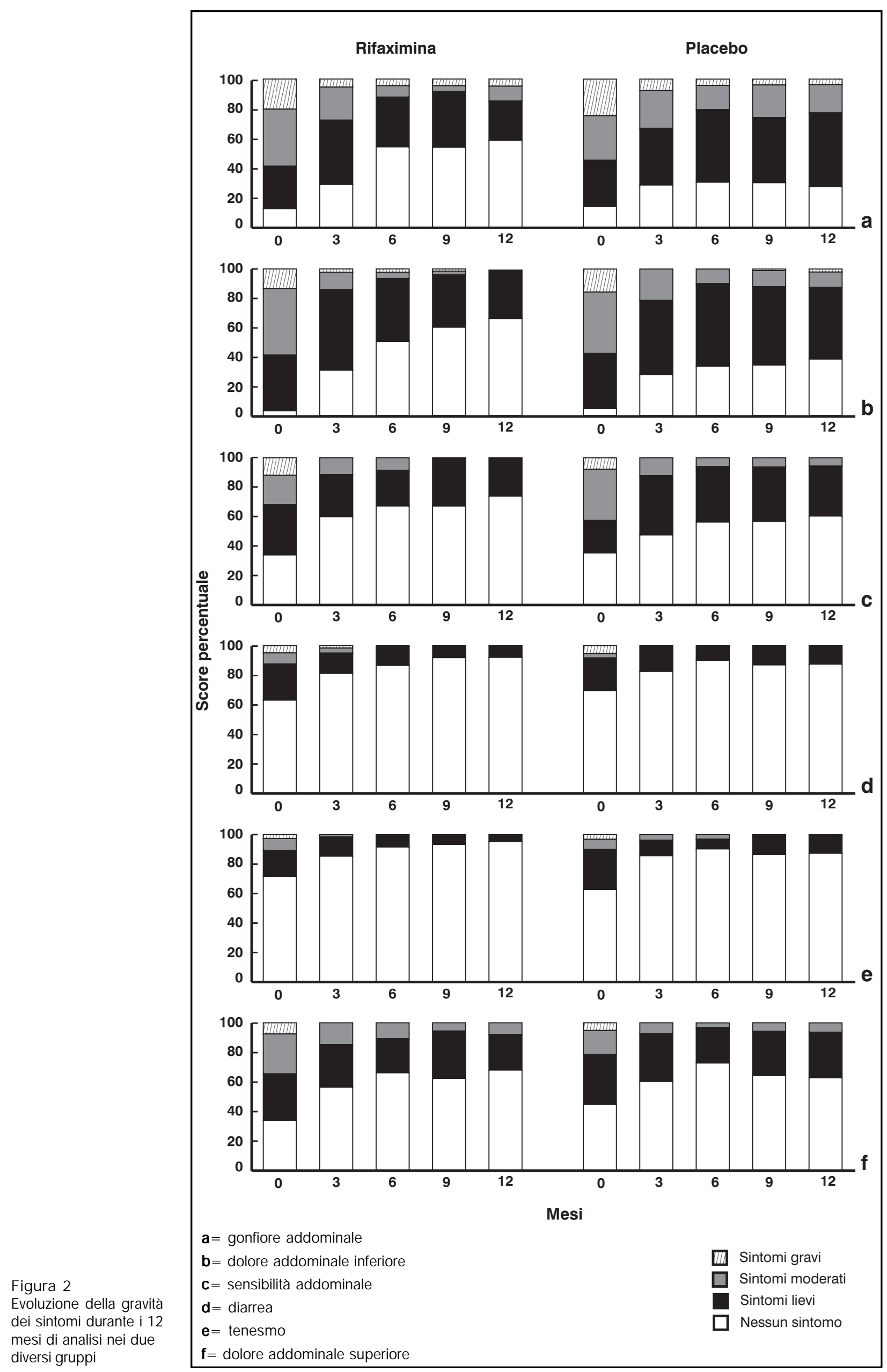


maggior riduzione di tale punteggio durante le valutazioni effettuate al sesto, nono e dodicesimo mese. Al termine dello studio circa il 68,9\% dei pazienti riceventi rifaximina erano liberi da sintomi, o presentavano una sintomatologia leggera, contro il 39,5\% del gruppo placebo.

La Figura 2 riporta le variazioni, nel corso dello studio, dei diversi sintomi in termini di gravità: il gonfiore addominale risulta significativamente ridotto in seguito alla terapia antibiotica già dal sesto mese in poi $(\mathrm{p}<0,01 \mathrm{a}$ sei mesi, $\mathrm{p}<0,001$ a nove e 12 mesi); nel gruppo trattato il dolore addominale inferiore mostra una diminuzione che, rispetto al gruppo placebo, diviene statisticamente significativa dal nono mese ( $\mathrm{p}<0,01$ a nove mesi, $\mathrm{p}<0,001$ a 12 mesi), mentre per quanto riguarda la riduzione della sensibilità addominale la differenza tra i due gruppi acquista significatività al dodicesimo mese ( $\mathrm{p}<0,05$ a 12 mesi).

La notevole riduzione del gonfiore e del dolore addominale inferiore rilevate in seguito alla terapia antibiotica confermano l'ipotesi che la microflora intestinale giochi un ruolo fondamentale nella produzione di questi disagi. L'efficacia a lungo termine di rifaximina nell' alleviare i sintomi correlati alla malattia diverticolare è stata ulteriormente studiata da un recente studio multicentrico condotto da Latella e colleghi [34]; il trial, condotto in un vasto campione di pazienti, aveva inoltre come obiettivo secondario quello di misurare l'incidenza di episodi di diverticolite nel gruppo randomizzato a rifaximina rispetto al gruppo di controllo.

Le caratteristiche e i risultati dello studio sono riassunti in Tabella II.

Lo score globale è la somma dei punteggi assegnati, mediante questionario e visita clinica quadrimestrale, ai diversi sintomi: lo studio rileva una riduzione di tale parametro in entrambi i gruppi, ma fra i pazienti trattati con rifaximina la diminuzione è risultata significativamente maggiore.

Terminati i 12 mesi di trattamento, fra i pazienti riceventi rifaximina, la percentuale di pazienti privi di sintomatologia è stata significativamente superiore al gruppo di controllo.
Come si può osservare dai dati riportati in Tabella II, rifaximina sembra essere anche in grado di diminuire l'incidenza delle comuni complicanze della malattia diverticolare.

Porta e colleghi hanno condotto uno studio retrospettivo proprio al fine di indagare il ruolo della terapia antibiotica nel prevenire la comparsa di tali complicanze, e più specificatamente la possibilità che, in seguito ad un episodio di diverticolite acuto, tale terapia possa ridurre il rischio di riospedalizzazione o di intervento chirurgico [35].

Gli autori hanno esaminato tutti i casi di ricovero avvenuti fra il 1967 il 1991 a causa di complicazioni della malattia diverticolare: tra $\mathrm{i}$ 505 pazienti rilevati, 350 sono stati dimessi con prescrizione medica relativa a terapia antibiotica (una settimana/mese di paromomicina, rifaximina o neomicina associata a bacitracina) e 155 sono stati dimessi senza alcuna prescrizione medica (gruppo controllo).

Il ricovero era avvenuto in seguito allo sviluppo di complicanze, quali occlusione intestinale $(51,7 \%)$, perforazione $(23,3 \%)$ ed emorragia $(23,8 \%)$; una piccola percentuale di pazienti era stato ricoverato a causa dello sviluppo di fistole.

Il problema venne risolto chirurgicamente in 77 casi, di cui 15 in emergenza, per un rischio totale di essere sottoposti ad operazione del 15,2\%.

Per il 6\% $(n=22)$ dei pazienti dimessi con prescrizione si rese necessaria la riospedalizzazione e di questi il 14\% $(n=3)$ fu sottoposto ad intervento chirurgico; nel gruppo controllo invece il secondo ricovero fu necessario per il $12 \%$ dei pazienti $(n=19)$, e nel $53 \%(\mathrm{n}=10)$ dei casi la risoluzione delle complicazioni fu chirurgica.

In totale, su 505 pazienti dimessi, 41 vennero riospedalizzati per cause inerenti alla malattia diverticolare (diverticolite 34 casi, stenosi 5 casi, perforazione intestinale 2 casi).

Sulla base di questi dati, Porta e colleghi, hanno calcolato la riduzione assoluta di rischio (RAR) intesa come la differenza $\left(\mathrm{P}_{\mathrm{t}}-\mathrm{P}_{\mathrm{c}}\right)$ fra il tasso di riospedalizzazioni, o di risoluzioni chirurgiche, della frazione di pazienti trattati con antibiotici $\left(\mathrm{P}_{\mathrm{t}}\right)$ e quello della frazione di pazienti senza prescrizione $\left(\mathrm{P}_{\mathrm{c}}\right)$.

\begin{tabular}{|c|c|c|c|c|c|c|}
\hline Evento & $\begin{array}{c}\text { Eventi } \\
\text { pazienti t }\end{array}$ & $P_{t}$ & $\begin{array}{c}\text { Eventi } \\
\text { pazienti c }\end{array}$ & $P_{c}$ & $\begin{array}{c}\text { RAR } \\
\left(P_{t}-P_{c}\right)\end{array}$ & $\begin{array}{c}\text { RRR } \\
\left(P_{t}-P_{c} / P_{c}\right)\end{array}$ \\
\hline Riospedalizzazione & $22 / 350$ & $6 \%$ & $19 / 155$ & $12 \%$ & $6 \%$ & $50 \%$ \\
\hline Intervento chirurgico* & $3 / 22$ & $14 \%$ & $10 / 19$ & $53 \%$ & $39 \%$ & $73 \%$ \\
\hline
\end{tabular}

Tabella III Valori di RAR e di RRR calcolati da Porta e colleghi [35]

\footnotetext{
* Le percentuali riportate si riferiscono ai pazienti riospedalizzati e non ai pazienti totali appartenenti ad ognuno dei gruppi
} 
È stata altresì calcolata la riduzione relativa di rischio (RRR) espressa come la riduzione assoluta di rischio proporzionata al tasso di riospedalizzazioni, o di risoluzioni chirurgiche, del gruppo di controllo $\left(\mathrm{P}_{\mathrm{t}}-\mathrm{P}_{\mathrm{c}} / \mathrm{P}_{\mathrm{c}}\right)$.

Come si evince dalla Tabella III, nei pazienti che, in seguito a un episodio acuto della patologia, si sono sottoposti ad una terapia antibiotica, il rischio di incorrere in complicazioni tali da rendere necessaria la riospedalizzazione o la risoluzione chirurgica è diminuito notevolmente.

Significativi miglioramenti nella sintomatologia e nella regolazione delle abitudini intestinali, in seguito al trattamento con rifaximina di pazienti affetti da malattia diverticolare non complicata, sono stati riportati da numerosi altri studi [5,36,37]; inoltre è stata dimostrata la capacità della terapia antimicrobica di determinare, probabilmente riducendo la degradazione batterica delle fibre, un aumento della massa fecale con conseguente diminuzione della compattezza delle feci e riduzione della pressione intralumare [29].

\section{Tollerabilità}

Nella maggior parte degli studi analizzati $[5,11,30,33,34,35,37,38]$ non sono riportati particolari effetti collaterali legati all'utilizzo di rifaximina; questo risultato è in accordo con i dati presentati da Gillis e colleghi, i quali hanno raccolto e analizzato 30 trial clinici, per un totale di 963 pazienti e 18 volontari sani, in cui era stata valutata la tollerabilità del farmaco.

Gli autori di questi studi raramente hanno rilevato reazioni avverse a carico di rifaximina e, nei casi in cui questo si è verificato, tali reazioni sono state soprattutto di natura gastrointestinale: cinque pazienti hanno riportato flatulenza, due dolore addominale [39], uno nausea [40] e due pazienti in età pediatrica hanno riportato episodi di vomito [41]. In tutti i casi, l'effetto indesiderato si è manifestato con un incidenza minore dell' $1 \%$.

Nei pazienti affetti da encefalopatia epatica, rifaximina ha dimostrato di essere meglio tollerata sia del lattulosio $[14,39,41,42]$ che della neomicina [43].

Dopo il trattamento con rifaximina è stato spesso riportato un aumento dei livelli sierici di potassio $[44,45]$ e di sodio $[42,44]$, ma senza oltrepassare il limite di normalità.

Nell'insieme il farmaco ha mostrato un ottimo profilo di sicurezza, dovuto essenzialmente alla sua scarsissima assorbibilità che ne rende pressoché nulli gli effetti sistemici.

\section{CONSIDERAZIONI FARMACOECONOMICHE}

L'incidenza della malattia diverticolare nei paesi sviluppati è aumentata drammaticamente nel corso degli ultimi decenni e sembra essere strettamente collegata al consumo sempre più diffuso di cibi raffinati e alla concomitante riduzione della quantità di fibre assunte con la dieta [18].

Alcuni studi epidemiologici [46-48] hanno dimostrato che la prevalenza di questa patologia differisce notevolmente da uno stato all'altro; in Italia, conformemente a ciò che succede nel resto d'Europa, la prevalenza di questo disturbo aumenta regolarmente con l'età e oltre i 60 anni la sua progressione sembra essere più rapida: circa il $5 \%$ della popolazione è colpito da diverticolosi nella quarta decade di vita e circa il 40-60\% superati i 70 anni [6].

Negli Stati Uniti, in Australia e in altri paesi industrializzati questo disturbo colpisce in media circa il $10 \%$ della popolazione [49-51].

Fra gli occidentali, la prevalenza risulta leggermente minore fra i vegetariani e fra coloro che introducono una grande quantità di fibre con la dieta [52]; la diverticolosi è quasi sconosciuta nell' Africa rurale e in Asia (eccetto per quanto riguarda il Giappone in cui la prevalenza risulta simile a quella riscontrabile negli Stati Uniti).

I dati presenti in letteratura sulla diffusione della patologia possono a volte essere imprecisi a causa del fatto che nella maggioranza dei casi la malattia diverticolare è asintomatica: solo una percentuale di pazienti compresa fra il 10 e il $25 \%$ sviluppa qualche sintomo nel corso della vita [53].

La malattia diverticolare presenta una serie di implicazioni socioeconomiche, non solo per la sua elevata incidenza, ma anche per le limitate conoscenze sulla storia naturale della patologia e sui fattori che aumentano il rischio di sviluppare complicanze: questo comporta notevoli difficoltà nel definire una strategia preventiva efficace.

Negli Stati Uniti, secondo un rapporto redatto dall' American Gastroenterological Association sui costi relativi alle patologie dell'apparato gastrointestinale, la malattia diverticolare, con un costo annuale di 2,6 miliardi di dollari, rappresenta il quinto disturbo più oneroso in termini di costi diretti e indiretti [54].

Al fine di analizzare le implicazioni sociali e l'impatto economico della malattia diverticolare, un gruppo di ricercatori inglesi ha analizzato in modo retrospettivo evoluzione clinica e costi sostenuti per le visite, il trattamento e l'ospedalizzazione di 148 pazienti, 
prendendo come periodo di riferimento l'anno finanziario.

Fra i pazienti presi in esame 83 sono stati ricoverati (per più di un giorno); di questi 55 sono stati ricoverati d'urgenza e 5 sono deceduti (6\% dei pazienti ospedalizzati). Un anno dopo essere stati dimessi, 134 pazienti su 148 erano in vita $(90,5 \%)$.

I ricercatori hanno calcolato che, durante l'anno considerato, il numero totale di giorni di ricovero è stato pari a 982, di cui 94 nel reparto di terapia intensiva; sono state eseguite 19 operazioni chirurgiche, di cui 16 colectomie, e 410 visite specialistiche.

Le indagini cliniche hanno richiesto lo svolgimento di 48 colonscopie, 77 sigmoidoscopie flessibili, 77 clismi con bario e alcuni altri esami.

Il costo totale calcolato nello studio è risultato essere pari a 465.263 sterline (corrispondente al 5,3\% del budget annuale destinato alla chirurgia generale). Il $70 \%$ del costo totale è rappresentato dalle spese di ospedalizzazione, di cui quelle relative al reparto di terapia intensiva ne costituiscono più di un terzo [55].

Le complicazioni della malattia diverticolare che più frequentemente sono responsabili del ricovero in ospedale sono diverticolite $(76,3 \%)$ ed emorragia (13,3\%) [56]; il 90\% dei casi di emorragia e il $75 \%$ dei casi di diverticolite non sono trattati chirurgicamente e il tasso totale di mortalità è dell' $11,3 \%$. Uno studio inglese ha invece rilevato fra i pazienti ricoverati con malattia diverticolare complicata una percentuale di interventi chirurgici del $31,7 \%$ e un tasso di mortalità post-operatoria del 12\% [57].

Il tasso di mortalità risulta comunque molto diverso a seconda che l'operazione sia eseguita in emergenza (17\% circa) o in elezione (1$4 \%)[58,59]$.

Negli Stati Uniti il tasso di ospedalizzazione è di circa 238/100.000 se si considerano pazienti di età compresa fra 65 e 69 anni, mentre sale a 631/100.000 fra i pazienti con un età superiore a 85 anni [60]; il tasso di mortalità risulta di 2,5/100.000.

Nel 2002 in Italia, su 935.113 ricoveri per malattie dell'apparato digerente, 31.562 pazienti hanno riportato come diagnosi primaria quella di diverticolite o diverticolosi; in questi casi la durata media di degenza è stata di 9,17 giorni [61].

Riuscire ad evitare l'infiammazione dei diverticoli, la loro perforazione o la formazione di una fistola significa evitare ospedalizzazioni e interventi chirurgici che naturalmente, oltre ad avere un impatto economico elevatissimo, aumentano anche il rischio di mortalità. Risulta pertanto di notevole importanza diagnosticare e trattare tempestivamente anche le forme lievi, ambulatoriali, con intenti di prevenzione secondaria.

\begin{tabular}{lcc}
\hline \multicolumn{1}{c}{ Descrizione } & DRG & Tariffa \\
\hline Diverticolosi senza complicanze & M-183 & 1.197 euro \\
Diverticolosi con complicanze & M-182 & 2.088 euro \\
Sigmoidectomia senza complicanze & C-149 & 5.792 euro \\
Sigmoidectomia con complicanze & C-148 & 10.396 euro \\
\hline
\end{tabular}

$M=$ medico $C=$ chirurgico

Tabella IV

Tariffe DRG per ricovero ordinario

Il consumo di risorse diviene ancora più imponente quando la malattia diverticolare complicata richiede una risoluzione chirurgica. Un gruppo di ricercatori statunitensi ha raccolto una serie di dati relativi a pazienti affetti da diverticolite che, fra l'inizio del 1999 e la fine del 2000, sono stati sottoposti a resezione del tratto sigmoide del colon [62]. I costi relativi alle 132 colectomie effettuate, svolte a cielo aperto $(n=71)$ o in laparoscopia $(n=61)$, sono stati calcolati mediante l'utilizzo di un software specializzato nella gestione dei costi ospedalieri. La durata media della degenza è stata di 3,1 giorni per i pazienti operati in laparoscopia e di 6,8 per quelli operati in laparotomia ed entrambi i gruppi hanno manifestato casi di infezione della ferita o complicazioni polmonari (anche se in percentuale minore nel caso di intervento laparoscopico). La riospedalizzazione si è resa necessaria nel 4,9\% dei pazienti operati in laparoscopia e nel 5,6\% dei pazienti operati a cielo aperto, mentre un solo paziente è deceduto durante l'operazione (intervento laparoscopico). Il costo medio unitario è risultato di 3.458 e di 4.321 dollari, rispettivamente per i pazienti operati in laparoscopia e per quelli operati a cielo aperto.

Nonostante la maggior costo/efficacia del primo tipo di intervento rispetto al secondo, il consumo di risorse legato al trattamento chirurgico della diverticolite, la più comune tra le complicanze della diverticolosi, risulta molto oneroso.

Per avere un'idea dell' ordine di grandezza di tali spese in relazione al nostro Paese, riportiamo in Tabella IV le tariffe rimborsate dal SSN in alcuni casi di ricovero o intervento chirurgico legati alla malattia diverticolare, calcolate secondo i DRG attualmente in vigore per la regione Piemonte.

Secondo alcuni studi riportati precedentemente $[34,35]$ la terapia antibiotica sarebbe in grado di diminuire significativamente il rischio 
Figura 3

Stima dei costi annuali relativi a due ipotetici gruppi di pazienti di cui uno trattato con rifaximina e l'altro non trattato con antibiotici (*costi in euro)

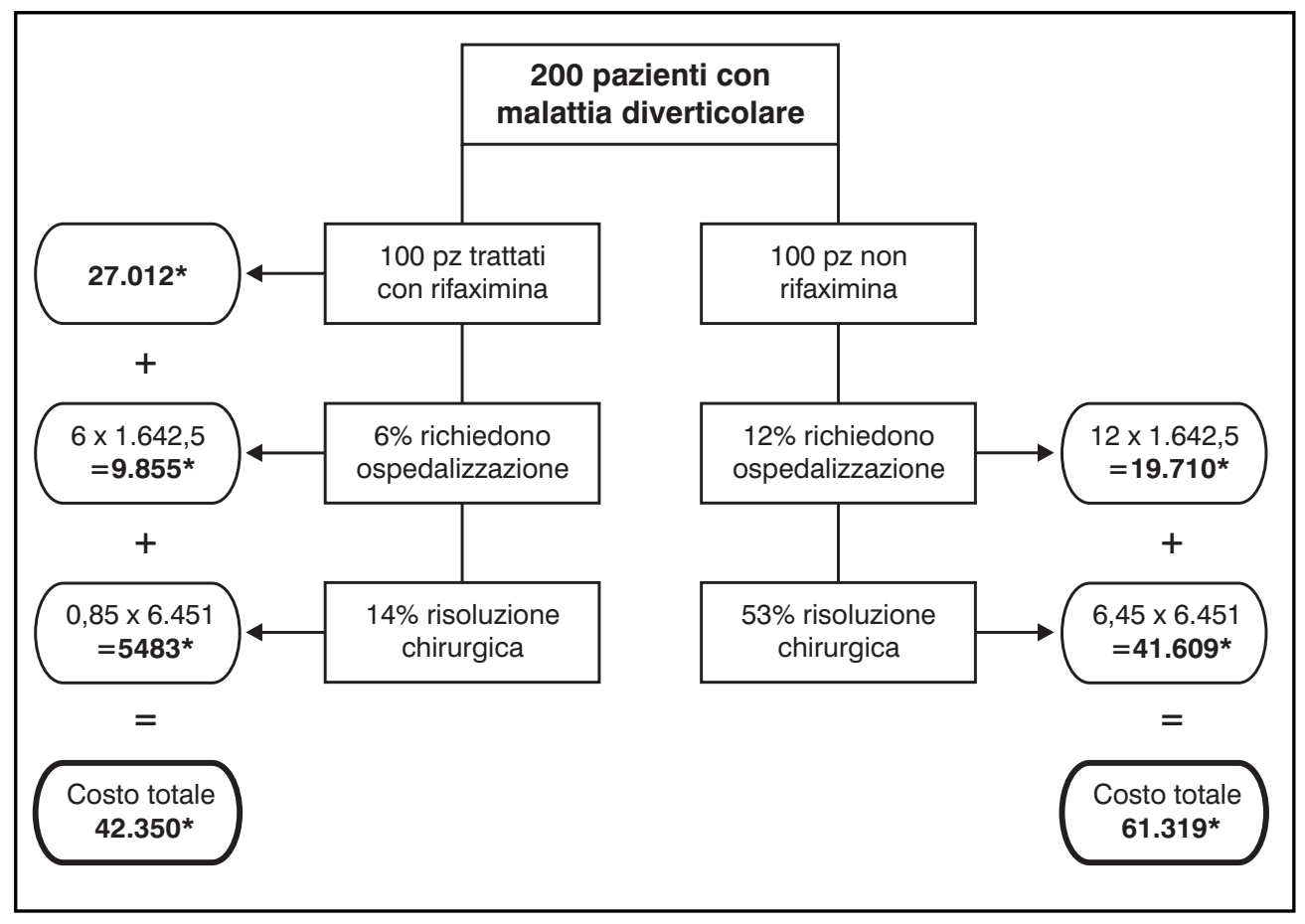

per il paziente di essere sottoposto ad intervento chirurgico (o ad ospedalizzazione): con l'uso di tale terapia questi costi (DRG) si potrebbero in parte tramutare in risparmi.

Ovviamente anche questi farmaci hanno un costo: una confezione di 12 compresse da 200 mg di rifaximina (Normix ${ }^{\circledR}$ - Alfa Wassermann) costa 9,65 euro; se si considera una dose di $800 \mathrm{mg} /$ die per sette giorni al mese (posologia utilizzata nei trial analizzati) il costo mensile del farmaco risulta pari a 22,51 euro, che significa un costo annuale di 270,12 euro.

Il prezzo contenuto, oltre al fatto che la sua assunzione non richiede particolari spese di somministrazione, di management degli effetti collaterali o di monitoraggio dei livelli plasmatici, rende la terapia economicamente vantaggiosa, soprattutto in vista dei suddetti costi evitati (risparmio sui ricoveri e sulle spese chirurgiche).

Al fine di stimare la dimensione di tale risparmio, calcoliamo i costi relativi al trattamento di due ipotetici gruppi di pazienti, uno ricevente rifaximina e l'altro no (Figura 3); per effettuare tale computo assumiamo che le percentuali di pazienti sottoposti a ricovero o a intervento chirurgico siano quelle rilevate nei due gruppi da Porta e colleghi [35].

Come costo di ricovero è stato utilizzato il valore medio (1.642 euro) fra il DRG relativo a diverticolosi senza complicanze e con complicanze; lo stesso procedimento è stato adottato per la determinazione del costo chirurgico (8.094 euro). La differenza tra questi due valori (6.451 euro) rappresenta la spesa in più per ogni paziente che viene sottoposto anche ad intervento chirurgico.

Il risparmio annuo per un gruppo di 100 pazienti risulta di circa 20.000 euro; occorre però specificare che la flow chart rappresenta solo una stima ipotetica dei costi e che in essa sono stati considerati solo i costi sanitari diretti, tralasciando per ragioni pratiche sia quelli indiretti che quelli intangibili. Il ricovero induce infatti un costo da mancato guadagno che grava sia sul paziente che sulla società: nel caso di diverticolosi o diverticolite la degenza ha una durata media di 9,17 giorni, ma essa può raggiungere anche i 20 giorni in caso di intervento chirurgico [61].

La capacità della rifaximina di ridurre, e in qualche caso eliminare, la sintomatologia correlata alla malattia diverticolare e, soprattutto, di diminuire il rischio di ospedalizzazione potrebbe ridurre notevolmente tali costi (mancato guadagno); inoltre il farmaco, grazie a questi effetti, permetterebbe di migliorare lo stato psicologico e sociale del paziente.

Quest'ultimo aspetto, legato alla qualità di vita dei pazienti, merita particolare attenzione in considerazione della sempre maggiore influenza che esso ha sulle scelte compiute da paziente e medico in tema di management della patologia.

Un primo studio a tale proposito è stato condotto nel 2002 da un team di ricercatori inglesi [63], i quali hanno sottoposto ad un questionario sulla qualità di vita un gruppo formato da 50 pazienti affetti da malattia diverticolare sintomatica (15 dei quali precedentemente ri- 
coverati per peggioramento del quadro clinico) e un gruppo di controllo formato da 50 volontari sani. Il questionario era costituito da 10 domande relative ai sintomi specifici della malattia, 5 relative ai sintomi sistemici e 17 riguardanti le funzioni emozionali (12) e sociali (5); la qualità di vita veniva quindi espressa mediante la somma degli score assegnati alle singole domande (punteggi da 1 a 7).

Per valutare l'appropriatezza del metodo e dei temi indagati, il questionario (prima di essere presentato a pazienti e gruppo controllo) è stato approvato da un panel di esperti costituito principalmente da chirurghi, infermieri e assistenti dell'unità colonproctologica.

Come riportato in Tabella V, i dati ricavati dal questionario indicano un dislivello significativo dello score medio relativo ai pazienti affetti dalla patologia rispetto allo score ottimale e, in particolare, rispetto al gruppo dei volontari sani; questo si osserva in tutte e quattro le categorie esaminate e, indubbiamente, è indice dell'impatto fortemente negativo della sintomatologia sulla qualità di vita dei pazienti.

La capacità dimostrata da rifaximina $[11,30,33]$ nel ridurre notevolmente la gravità della sintomatologia e nel rendere una buona percentuale di pazienti liberi da sintomi potrebbe quindi concretizzarsi in un notevole miglioramento della qualità della vita.

Per il paziente tale miglioramento risulta ancora più significativo nel caso in cui si riesca ad evitare la comparsa di complicazioni; alcune di esse, come per esempio le fistole colovaginali [64], raramente sono curabili mediante terapia farmacologica; nella maggior parte dei casi esse richiedono una risoluzione chirurgica e non sempre si riesce a ripristinare la continuità intestinale: questo, oltre ad abbassare decisamente il livello di qualità di vita, aumenta il rischio di mortalità.

In numerosi casi di sanguinamento del colon o attacchi ricorrenti di diverticolite si rende necessario praticare una colectomia totale con anastomosi ileo-rettale; questa nuova condizione è accompagnata, in particolare nel periodo immediatamente dopo l'intervento, da debilitanti scariche di diarrea e incontinenza con un impatto fortemente negativo sullo stile di vita e sulle funzioni lavorative e sociali $[65,66]$; inoltre è stato dimostrato da uno studio condotto a Singapore che, anche in quei pazienti che in seguito all'intervento mostrano una frequenza di evacuazione e una continenza abbastanza normali, il livello di qualità di vita è comunque danneggiato dal continuo timore del verificarsi dei sintomi sopracitati [67]. Per i pazienti che hanno subito una colostomia, la situazione in

\begin{tabular}{|c|c|c|c|}
\hline \multirow{2}{*}{ Aree analizzate } & \multicolumn{3}{|c|}{ Score QoL medio } \\
\hline & Ottimale & Controllo & Pazienti \\
\hline Sintomi addominali & 70 & 64,3 & 43,8 \\
\hline Sintomi specifici & 35 & 29,1 & 21,1 \\
\hline Funzioni emozionali & 84 & 74,1 & 55,5 \\
\hline Funzioni sociali & 35 & 33,4 & 24,3 \\
\hline
\end{tabular}

Tabella $\mathrm{V}$

Differenze statisticamente significative fra i punteggio $Q$ oL dei due gruppi analizzati [63]

relazione alla propria qualità di vita, risulta, ovviamente, ancora più delicata [68].

Per tali ragioni risulta di fondamentale importanza cercare la migliore strategia di prevenzione: utilizzare un farmaco in grado di ridurre il rischio di complicanze della malattia diverticolare significa a lungo termine risparmiare sui costi e mantenere alto il livello di qualità di vita del paziente e dei suoi famigliari.

Paragonato all' ampia scelta di antibiotici sistemici in commercio, il numero di antibiotici intestinali, utilizzabili in particolari disturbi del tratto digestivo, è estremamente ristretto e il loro uso è spesso limitato agli aminoglicosidi, in particolare alla paromomicina e alla neomicina. Quest'ultima tuttavia è assorbita dal tratto gastrointestinale in misura pari al $3 \%$ della dose somministrata [12], quantità che in certe condizioni può determinare effetti collaterali che, soprattutto nel caso di uso prolungato ad elevati dosaggi, possono limitarne l'uso nella pratica clinica. Quelli riferiti con maggiore frequenza sono nausea, vomito e stipsi; durante le terapie prolungate o ripetute può verificarsi una sindrome da malassorbimento con diarrea e steatorrea verosimilmente legata a inibizione delle lipasi, fenomeni di nefrotossicità (oliguria, albuminuria, ematuria, iperazotemia) e di ototossicità (vertigini, ronzii, ipoacusia) $[12,69]$.

La paromomicina, somministrata oralmente, ha un assorbimento attraverso l'apparato digerente quasi nullo; quindi solo in casi di abnorme assorbimento, specie con dosi elevate o eccessive, sono stati riportati fenomeni di nefrotossicità $\mathrm{o}$ di ototossicità.

Il management degli effetti collaterali può rappresentare un costo anche abbastanza oneroso e, specie nel caso di terapie a lungo termine, può comportare un notevole peggioramento della qualità di vita. 
Al contrario di rifaximina che, come detto precedentemente, possiede un ampio spettro d'azione, il limite più importante degli aminoglicosidi resta quello di avere uno spettro antibatterico troppo ristretto per essere usato con successo in patologie causate da diverse specie batteriche: essi hanno sui batteri gram-positivi e sui batteri anaerobi un effetto molto limitato [12,70]. Per tali ragioni spesso l'uso di neomicina è associato a quello di bacitracina, che è invece attiva contro i batteri gram-positivi.

Un altro problema legato all'utilizzo cronico di tali antibiotici è la comparsa di fenomeni di resistenza che si instaurano frequentemente a causa di enzimi prodotti dal microrganismo che inattivano il farmaco; per quanto concerne gli aminoglicosidici tale resistenza, essendo di tipo plasmidico, si trasmette orizzontalmente da cellula a cellula costituendo un grosso problema clinico ed epidemiologico. La resistenza nei confronti di rifaximina è invece di tipo cromosomico, quindi trasmissibile solo tramite discendenza.

In letteratura non sono presenti studi clinici prospettici in cui sia stata analizzata l'efficacia di neomicina, o di paromomicina, nel ridurre la sintomatologia o il rischio di complicazioni in pazienti affetti da malattia diverticolare, e soprattutto in cui l'azione di rifaximina sia stata confrontata direttamente con questi due farmaci.

La loro efficacia è stata però confrontata in relazione ad altre patologie come le infezioni intestinali con diarrea, l'encefalopatia epatica o la profilassi prechirurgica.

In alcuni trial, l'efficacia di rifaximina nel ridurre il livello plasmatico di ammonio in pazienti affetti da encefalopatia epatica è risultata maggiore di quella di neomicina $[42,43]$, in altri è risultata simile [71].
Nel trattamento della diarrea indotta da infezione batterica, neomicina e rifaximina hanno dato risultati molto simili sia come attività che come tempo impiegato per normalizzare le funzioni intestinali [72-75]; in uno studio condotto su pazienti pediatrici però, rifaximina è stata completamente tollerata mentre sono stati riportati due casi di forte dolore allo stomaco nel gruppo trattato con neomicina in associazione a bacitracina $(n=17)$ [76].

Sempre su pazienti in età pediatrica affetti da diarrea batterica, è stato svolto uno studio al fine di valutare l'efficacia di rifaximina contro paromomicina: i risultati ottenuti con rifaximina sono stati migliori di quelli ottenuti con paromomicina (sebbene senza raggiungere la significatività statistica) e soprattutto si sono ottenuti più velocemente [77].

Nel complesso rifaximina ha dimostrato di essere efficace almeno come gli altri due antibiotici e, in alcuni casi, meglio tollerata.

Per avere un'idea del costo delle diverse terapie prese in esame, riportiamo in Tabella VI il costo d'acquisto dei farmaci sopracitati (la dose e la durata del trattamento su cui ci si è basati per il calcolo sono state tratte dalla letteratura).

\section{CONCLUSIONI}

La terapia antibiotica è comunemente usata nel trattamento della diverticolite e delle altre complicanze della malattia diverticolare, mentre il suo utilizzo nella cura della patologia non complicata è ancora dibattuto. Tuttavia alcune osservazioni suggeriscono un possibile ruolo della microflora intestinale nel provocare la comparsa di alcuni sintomi correlati alla patologia: il gas prodotto dal metabolismo batterico potrebbe essere la causa di sintomi come il gonfiore e il dolore addominale e l'azio-
Tabella VI

Costo d'acquisto del farmaco riferito ad un mese di terapia

\begin{tabular}{|c|c|c|c|}
\hline Farmaco & Costo confezione & Posologia & Costo mensile \\
\hline Neomicina/bacitracina & $\begin{array}{l}16 \text { compresse neomicina } \\
\text { solfato F.U. } 25.000 \text { U.I. } \\
\text { bacitracina F.U. } 2.500 \text { U.I. } \\
\text { 8,40 euro (Bimixin } \AA \text { ) }\end{array}$ & $\begin{array}{c}\text { neomicina } \\
150.000 \cup \mathrm{I} / \text { die } \\
\text { bacitracina } \\
15.000 \cup \mathrm{I} / \text { die } \\
\text { per } 7 \mathrm{gg} / \mathrm{mese}\end{array}$ & 22,05 euro \\
\hline Paromomicina & $\begin{array}{c}16 \text { capsule paromomicina } \\
250 \mathrm{mg} \\
\left.11,6 \text { euro (Humatin }{ }^{\circledR}\right)\end{array}$ & $\begin{array}{c}\text { paromomicina } \\
1,5 \mathrm{~g} / \mathrm{die} \\
\text { per } 7 \mathrm{gg} / \mathrm{mese}\end{array}$ & 30,45 euro \\
\hline Rifaximina & $\begin{array}{c}12 \text { compresse rifaximina } \\
200 \mathrm{mg} \\
\left.\text { 9,65 euro (Normix }{ }^{\circledR}\right)\end{array}$ & $\begin{array}{c}\text { rifaximina } \\
800 \mathrm{mg} / \mathrm{die} \\
\text { per } 7 \mathrm{gg} / \mathrm{mese}\end{array}$ & 22,51 euro \\
\hline
\end{tabular}


ne degradante dei batteri nei confronti delle fibre potrebbe rendere la massa fecale più dura e compatta, aumentando così la pressione intraluminale.

Questa ipotesi è confermata dal fatto che numerosi trial clinici hanno dimostrato che il trattamento ciclico di rifaximina è più efficace rispetto alla sola somministrazione di fibre nell'alleviare i sintomi e nel prevenire le più comuni complicanze della patologia diverticolare.

La rifaximina è il derivato non assorbibile della rifamicina, di cui mantiene l'ampio spettro d'azione comprendente anche $\mathrm{i}$ batteri anaerobi: questa è una caratteristica fondamentale, considerato che l'uso di antibiotici sistemici, a causa dei loro effetti collaterali, dovrebbe essere limitato ai casi di diverticolosi complicata, e gli enteroantibiotici solitamente hanno uno spettro d'azione ristretto.

Grazie alla sua scarsissima assorbibilità (minore dell' $1 \%$ della dose somministrata) nessun effetto collaterale di rilievo è stato riportato dai pazienti nel corso dei trial presi in esame.

Oltre al miglioramento della qualità della vita che il farmaco può indurre, alleviando e in qualche caso eliminando la sintomatologia, diventa molto importante a livello economico l'azione preventiva che rifaximina può svolgere nel ridurre l'incidenza di complicanze della patologia: il suo utilizzo infatti, a fronte di un limitato costo d'acquisto, può permettere al Sistema Sanitario Nazionale di risparmiare nel lungo periodo onerose spese chirurgiche e di ospedalizzazione, provvedimenti spesso necessari in caso di diverticolite, sanguinamenti ed altre complicazioni della malattia diverticolare.

\section{BIBLIOGRAFIA}

1. Torsoli A et al. Diverticular disease of the colon: data relevant to management. Gastroenterol Internat 1991; 4: 3-20.

2. Haglund $\mathrm{U}$ et al. Complicated diverticular disease of the sigmoid colon. An analysis of short and long term outcome in 392 patients. Ann Chir Gynaecol. 1979;68(2):41-6.

3. Parks TG, Connell AM. The outcome in 455 patients admitted for treatment of diverticular disease of the colon. Br J Surg. 1970 Oct;57(10):775-8.

4. Barbara $\mathrm{G}$ et al. A role for inflammation in irritable bowel syndrome? Gut. 2002 Jul;51 Suppl 1:i41-4.

5. Iosca N, Ferrieri A. The rifaximin therapy and prophylaxis of episodes of acute diverticulitis Recenti Prog Med. 1993 Jan;84(1):49-53. Italian.

6. Delvaux M. Diverticular disease of the colon in Europe: epidemiology, impact on citizen health and prevention. Aliment Pharmacol Ther. 2003 Nov; 18 Suppl 3:71-4.

7. Brodribb AJM. Treatment of symptomatic diverticular disease with a hich fiber diet. Lancet 1977; i: 664-666.

8. Ewerth $\mathrm{S}$ et al. Influence on symptoms and transit-time of Vi-SiblinR in diverticular disease. Acta Chir Scand Suppl. 1980;500:49-50.

9. Soltoft JL et al. Adouble blind trial of the effect of wheat bran on symptoms of irritable bowel syndrome. Lancet 1976; i: 270-272.

10. Longstreth GF et al. Psyllium therapy in the irritable bowel syndrome. A double-blind trial. Ann Intern Med. 1981 Jul;95(1):53-6.

11. Lombardo L, Lapertosa G. The ambulatory medical treatment of colonic diverticulitis. An open clinico-endoscopichistological study with rifaximin, a nonaminoglycoside enteric antibiotic. Recenti Prog Med. 1991 May;82(5):3004. Italian.

12. Di Febo G, Calabrese C, Matassoni F. New trends in non-absorbable antibiotics in gastrointestinal disease. Ital J Gastroenterol. 1992 Nov-Dec;24(9 Suppl 2):10-3. Review.

13. Mandell GL, Sande MA. Antimicrobial agents. In: Gilman AG et al editors. The pharmacological basis of therapeutics. $8^{\text {th }}$ ed. v.2 Singapore: McGraw-Hill Inc., 1992: 1146- 64.

14. Fera $\mathrm{G}$ et al. Rifaximin in the treatment of hepatic encephalopathy. Eur J Clin Res 1993. 4: 57-66.

15. NORMIX® - Riassunto delle caratteristiche del prodotto.

16. Hoover WW et al. Antimicrobial activity and spectrum of rifaximin, a new topical rifamycin derivative. Diagn Microbiol Infect Dis. $1993 \mathrm{Feb}$;16(2):111-8. 


\section{Rifaximina nel trattamento della malattia diverticolare: potenziale terapeutico ed economico}

17. Eftimiadi C, DeLeo C, Schito GC. Treatment of hepatic encephalopathy with L/105, a new non-absorbable rifamycin. Drugs Exp Clin Res 1984. 10: 691-6.

18. Gillis JC, Brogden RN. Rifaximin. A review of its antibacterial activity, pharmacokinetic properties and therapeutic potential in conditions mediated by gastrointestinal bacteria. Drugs. 1995 Mar;49(3):467-84.

19. DeLeo C, Eftimiadi C, Schito GC. Rapid disappearance from the intestinal tract of bacteria risistant to rifaximin. Drugs Exp Clin Res 1986. 12: 979-81.

20. Descombe JJ et al. Pharmacokinetic study of rifaximin after oral administration in healthy volunteers. Int J Clin Pharmacol Res. 1994;14(2):51-6.

21. De Angelis L. L-105. Drugs Future 1982. 7: 260-1.

22. Venturini AP. Pharmacokinetics of L/105, a new rifamycin, in rats and dogs, after oral administration. Chemotherapy. 1983;29(1):1-3.

23. Cellai L et al. Rifaximin (L/105), a new topical intestinal antibiotic: pharmacokinetic study after single oral administration of $3 H$-rifaximin to rats. Chemioterapia. $1984 \mathrm{Dec} ; 3(6): 373-7$.

24. Jiang $\mathrm{Z}$ et al. In vitro activity and fecal concentratrion of rifaximin after oral administration. Antimicrob Agents Chemother. 2000 Aug; 44(8):2205-6.

25. Miglio F et al. Rifaximin, a non-absorbable rifamycin, for the treatment of hepatic encephalopathy. A double-blind, randomised trial. Curr Med Res Opin. 1997;13(10):593-601.

26. Gionchetti $\mathrm{P}$ et al. Rifaximin in patients with moderate or severe ulcerative colitis refractory to steroid-treatment: a double-blind, placebo-controlled trial. Dig Dis Sci. 1999 Jun;44(6):1220-1.

27. Rizzello F et al. Rifaximin systemic absorption in patients with ulcerative colitis. Eur J Clin Pharmacol. 1998 Mar;54(1):91-3.

28. Engel RR, Levitt MD. Intestinal tract gas formation in newborns. In: Program for meeting of American Pediatric Society and Society for Pediatric Research, 1970: 266.

29. Kurpad AV, Shetty PS. Effects of antimicrobial therapy on faecal bulking. Gut. 1986 Jan;27(1):55-8.

30. Papi $\mathrm{C}$ et al. Efficacy of rifaximin on symptoms of uncomplicated diverticular disease of the colon. A pilot multicentre open trial. Diverticular Disease Study Group. Ital J Gastroenterol. 1992 Oct;24(8):452-6.

31. Ornstein $\mathrm{MH}$ et al. Are fibre supplements really necessary in diverticular disease of the colon? A controlled clinical trial. Br Med J (Clin Res Ed). 1981 Apr 25;282(6273):1353-6.

32. Hodgson WJ. The placebo effect. Is it important in diverticular disease? Am J Gastroenterol. 1977 Feb;67(2):157-62.

33. Papi $\mathrm{C}$ et al. Efficacy of rifaximin in the treatment of symptomatic diverticular disease of the colon. A multicentre double-blind placebo-controlled trial. Aliment Pharmacol Ther. 1995 Feb;9(1):33-9.

34. Latella $\mathrm{G}$ et al. Rifaximin improves symptoms of acquired uncomplicated diverticular disease of the colon. Int $\mathrm{J}$ Colorectal Dis. 2003 Jan;18(1):55-62.

35. Porta $\mathrm{E}$ et al. The natural history of diverticular disease of the colon: a role for antibiotics in preventing complications? A retrospective study. Riv Eur Sci Med Farmacol. 1994 Jan-Apr;16(1-2):33-9.

36. Ventrucci M et al. Evaluation of the effect of rifaximin in colon diverticular disease by means of lactulose hydrogen breath test. Curr Med Res Opin. 1994;13(4):202-6.

37. Giaccari S et al. Long-term treatment with rifaximin and lactobacilli in post-diverticulitic stenoses of the colon. Riv Eur Sci Med Farmacol. 1993 Jan-Feb;15(1):29-34.

38. Alvisi e D'ambrosi 1987 - Alvisi V et al. Rifaximin, a rifamycin derivative for use in the treatment of intestinal bacterial infections in seriously disabled patients. J Int Med Res. 1987 Jan-Feb;15(1):49-56.

39. Bucci L, Palmieri GC. Double-blind, double-dummy comparison between treatment with rifaximin and lactulose in patients with medium to severe degree hepatic encephalopathy. Curr Med Res Opin. 1993;13(2):109-18.

40. Salcuni PF, Palazzini E. An evaluation of the therapeutic activity and tolerance of rifaximin, administered by stomach probe in patients after colorectal surgery. Aggiornamenti Med Chir 1988. 6: 1-7.

41. Massa P, Vallerino E, Dodero M. Treatment of hepatic encephalopathy with rifaximin: double blind, double dummy study versus lactulose. Eur J Clin Res 1993. 4: 7-18.

42. Festi D et al. Rifaximinin in the treatment of chronic hepatic encephalopathy: results of a multicenter study of efficacy and safety. Curr Ther Res 1993. 54: 598-609. 
43. Pedretti $\mathrm{G}$ et al. Rifaximin versus neomycin on hyperammoniemia in chronic portal systemic encephalopathy of cirrhotics. A double-blind, randomized trial. Ital J Gastroenterol. 1991 May;23(4):175-8.

44. Luttichau U, Arcangeli P, Sinapi S. The use of Rifaximin in the treatment of acute diarrhoeal enteritis: open study. Panminerva Med. 1985 Jul-Sep;27(3):129-32.

45. Fiorentino $\mathrm{F}$ et al. Open study on the antidiarrhoeal effectiveness of the L 105 compound. Chemioterapia. 1984 Apr;3(2):132-5.

46. Painter NS, Burkitt DP. Diverticular disease of the colon: a deficiency disease of Western civilization. Br Med J. 1971 May 22;2(759):450-4.

47. Cheskin LJ, Lamport RD. Diverticular disease. Epidemiology and pharmacological treatment. Drugs Aging. 1995 Jan;6(1):55-63.

48. Vajrabukka T, Saksornchai K, Jimakorn P. Diverticular disease of the colon in a far-eastern community. Dis Colon Rectum. 1980Apr;23(3):151-4.

49. Naitove A, Almy TP. Diverticular disease of the colon. In: Sleisenger Fordtran ed. gastrointestinal disease. WB Saunders Company, 1989. 1419-1434.

50. Painter NS, Burkitt DP. Diverticular disease of the colon, a 20th century problem. Clin Gastroenterol. 1975 Jan;4(1):3-21.

51. Narasaka T et al. Statistical analysis of diverticulosis of the colon. Tohoku J Exp Med. 1975 Mar;115(3):271-5.

52. Gear JS et al. Symptomless diverticular disease and intake of dietary fibre. Lancet. 1979 Mar 10;1(8115):511-4.

53. Parks TG. Natural history of diverticular disease of the colon. Clin Gastroenterol. 1975 Jan;4(1):53-69.

54. AGA Announces Results of Landmark Study More than 283 Million Cases of Gastrointestinal Disease Cost at Least $\$ 42 *$ Billion Dollars Annually. The American Gastroenterological Association (www.gastro.org).

55. Papagrigoriadis S et al. Impact of diverticular disease on hospital costs and activity. Colorectal Dis. 2004 Mar;6(2):81-4.

56. Tudor RG, Farmakis N, Keighley MR. National audit of complicated diverticular disease: analysis of index cases. Br J Surg. 1994 May;81(5):730-2.

57. Sarin S, Boulos PB. Long-term outcome of patients presenting with acute complications of diverticular disease. Ann R Coll Surg Engl. 1994 Mar;76(2):117-20.

58. Rodkey GV, Welch CE. Colonic diverticular disease with surgical treatment. A study of 338 cases. Surg Clin North Am. 1974 Jun;54(3):655-74.

59. Levien DH et al. Safe resection for diverticular disease of the colon. Dis Colon Rectum. 1989 Jan;32(1):30-2.

60. Stollman et al. Hospitalisations for diverticular disease: effect of age on presentation. Am J Gastroenterol 1999; 11:3110-21.

61. Sito ufficiale del Ministero della Salute - www.ministerosalute.it.

62. Senagore AJ et al. Cost structure of laparoscopic and open sigmoid colectomy for diverticular disease: similarities and differences. Dis Colon Rectum. 2002 Apr;45(4):485-90.

63. Bolster LT, Papagrigoriadis S. Diverticular disease has an impact on quality of life - results of a preliminary study. Colorectal Dis. 2003 Jul;5(4):320-3.

64. Bahadursingh AM, Longo WE. Colovaginal fistulas. Etiology and management. J Reprod Med. 2003 Jul;48(7):489-95.

65. Eu KW et al. Clinical outcome and bowel function following total abdominal colectomy and ileorectal anastomosis in the Oriental population. Dis Colon Rectum. 1998 Feb;41(2):215-8.

66. Walsh RM, Aranha GV, Freeark RJ. Mortality and quality of life after total abdominal colectomy. Arch Surg. 1990 Dec;125(12):1564-6.

67. Lim JF, Ho YH et al. Total colectomy with ileorectal anastomosis leads to appreciable loss in quality of life irrespective of primary diagnosis. Tech Coloproctol. 2001 Aug;5(2):79-83.

68. Black PK. Psychological, sexual and cultural issues for patients with a stoma. Br J Nurs. 2004 Jun 24-Jul 7;13(12):692-7.

69. BIMIXIN® - Riassunto caratteristiche del prodotto.

70. Ripa S et al. In vitro antibacterial activity of rifaximin against Clostridium difficile, Campylobacter jejunii and Yersinia spp. Drugs Exp Clin Res. 1987;13(8):483-8.

71. Di Piazza S et al. Rifaximine versus neomycin in the treatment of portosystemic encephalopathy. Ital J Gastroenterol. 1991 Sep-Oct;23(7):403-7. 
72. Alvisi V et al. Treatment of secretory diarrhoeas. A double-blin trial of the effectiveness of rifaximin (L/105) and neomycin. Clin Trials Journal 1984; 21:215-223.

73. Palermo G, Di Gregorio P, Caffa G. Effectiveness of the L/105 compound in the treatment of acute diarrhoeal disease. A short term controlled study. Med Praxis 1984; 5: 147-152.

74. Mazzitelli et al. Antidiarrhoeal effectiveness and tolerance in man of local antibiotic: rifaximin. Comparative assessment using a control drug. Eur Rev Med Pharm Sci 1984; 6: 301-306.

75. Stornello C, Salanitri G. Contolled trial on the effectiveness of the new antidiarrheal drug rifaximin. Med Praxis 1987; 8: 69-78.

76. Beseghi U, De'Angelis GL. Comparison of two non-absorbable antibiotics for treatment of bacterial enteritis in children. Eur Rev Med Pharmacol Sci. 1998 May-Aug;2(3-4):131-6.

77. Frisari L, Viggiano V, Pelagalli M. An open, controlled study of two non-absorbable antibiotics for the oral treatment of paediatric infectious diarrhoea. Curr Med Res Opin 1997;14(1):39-45. 